\title{
Expression profile of cytokines in gastric cancer patients using proteomic antibody microarray
}

\author{
XIAOQIANG QUAN ${ }^{1,2}$, YI DING ${ }^{1}$, RUO FENG $^{1}$, XIAOYAN ZHU ${ }^{1}$ and QINXIAN ZHANG ${ }^{1}$ \\ ${ }^{1}$ Department of Histology and Embryology, Basic Medical College of Zhengzhou University, Zhengzhou, Henan 450001; \\ ${ }^{2}$ Department of Surgery, People's Hospital of Zhengzhou University, Zhengzhou, Henan 450003, P.R. China
}

Received June 3, 2017; Accepted September 5, 2017

DOI: $10.3892 / \mathrm{ol} .2017 .7104$

\begin{abstract}
Gastric cancer (GC) is often a deadly disease due to the late diagnosis and chemoresistance that characterizes many cases of this disease. The aim of this study was to explore a panel of candidate cytokines as diagnostic and predictive biomarkers for GC. Sixteen tissue samples of GC and adjacent noncancerous mucosa were selected from GC patients $(n=8)$ for antibody microarray analysis. Proteomic chip-based analysis was performed to simultaneously identify 507 cytokines using a cytokine antibody array in the gastric tissues to screen for differential proteins related in cases of GC. Fold changes of protein expression $>2.0$ or $<0.5$ were considered significant. The proteins that showed significant differences in levels between the cancerous and non-cancerous samples were analyzed using bioinformatics analysis. One hundred and five cytokines that were significantly different $(\mathrm{p}<0.05)$ between GC tissues and normal gastric mucosa were identified. Gene Ontology (GO) enrichment analysis showed that these differentially expressed proteins are involved in many biological and immunological processes, mainly in response to stress, chloroplast thylakoid membrane, vacuole, photosynthesis, aspartic-type endopeptidase activity and flavin adenine dinucleotide binding. Kyoto Encyclopedia of Genes and Genomes (KEGG) enrichment analysis indicated that these proteins mainly were involved in the process of cytokine-cytokine receptor interaction, transforming growth factor- $\beta$ (TGF- $\beta$ ) signaling pathway, pathways in cancer, tumor necrosis factor (TNF) signaling pathway, and mitogenactivated protein kinase (MAPK) signaling pathway. These findings suggest that the differentially expressed proteins could be associated with GC in patients. Further study of these cytokines may provide a promising approach for diagnosis, classification and prognosis of GC.
\end{abstract}

Correspondence to: Dr Xiaoyan Zhu or Dr Qinxian Zhang, Department of Histology and Embryology, Basic Medical College of Zhengzhou University, Zhengzhou, Henan 450001, P.R. China

E-mail: zhuxiaoyan8879@163.com

E-mail: qinxianzhang11@163.com

Key words: antibody microarray, proteomics, gastric cancer

\section{Introduction}

Gastric cancer (GC) is one of the common malignant tumors in human, ranking as the fourth most common cancer worldwide and the second highest cause of cancer-related death $(1,2)$. Currently, a combination of surgical resection and postoperative chemotherapy is the most common method of GC treatment. However, the majority of patients that manifest clinical symptoms are terminal as they already have metastasis and therefore, the overall survival rate of patients with GC is low. When GC is diagnosed at an early stage, its 5-year survival rate can be as high as $90 \%$. When diagnosed at a later stage, however, the 5-year survival rate of GC patients can be as low as $10 \%(3,4)$.

Biological markers have the potential to aid in early diagnosis, treatment and prognosis of tumors (5). In recent years, there have been some potential biomarkers that have emerged for the clinical diagnosis of GC, such as p27, cyclin E, E-cadherin, HER2, c-myc and p53. However, results have shown that there are no biomarkers that have been identified up-to-date that can be independently used for the occurrence and development of GC (6). Thus, developing molecular tagbased, sensitive and specific biomarkers for GC can have an important significance on the early diagnosis rate, effective treatment and reduced mortality of patients with GC.

When a tumor is present, cytokines and chemokines are produced by infiltrating inflammatory or tumor cells, which result in the change of the tumor microenvironment, and further promote tumor angiogenesis, proliferation, diffusion and metastasis (7). As there has been a flux of protein analysis technology, antibody microarray-based assays has been used for the research of disease proteomics, and it can comprehensively and accurately reflect the changes in protein expression levels that occur during the development of diseases $(8,9)$. Based on the changes of some upregulated or downregulated expression of proteins, antibody microarray-based technology can be used to discover biomarkers for early diagnosis, the evaluation of treatment effects and choice of new targets for treatment (10).

In this study, proteomic chip-based analysis was performed to simultaneously identify 507 cytokines using a cytokine antibody array in gastric tissues to screen for cytokines that were differentially expressed in cases of GC. We identified 105 cytokines with significant differences between GC tissues and normal gastric mucosa. Our results suggest that these differentially expressed cytokines could be associated with GC. 



Figure 1. Representative antibody-based array chips. Antibody-based array chips encompas 507 cytokines in duplicates probed with whole lysates from paired gastric cancer (GC) and non-GC mucosa in patients \#3 (A), \#5 (B), \#6 (C) and \#7 (D) (a1, b1, c1 and d1: adjacent noncancerous tissue; a2, b2, c2 and d2: gastric carcinoma tissue). The spot intensity of each protein signal was examined photometrically and normalized to the background noise in each spot of the negative controls. The spot intensity of each cytokine was merged and expressed as a mean value relative to the average signals of the positive controls on the protein array chip.

\section{Materials and methods}

Patients and tissue samples. Pairs of GC and adjacent noncancerous mucosa tissues were first diagnosed and confirmed by clinical and pathological examination. These samples were then obtained from patients $(n=8)$ who underwent D2 gastrectomy (radical gastrectomy with level 2 extended lymphadenectomy) between February, 2014 and June, 2015 at the Henan Provincial People's Hospital, Zhengzhou, China. The cancerous and normal gastric tissues were washed with physiological saline and subsequently frozen within 30 min of removal in a liquid nitrogen tank after immediate pathological examination. The senior pathologist routinely conducted the diagnosis for GC based on hematoxylin and eosin (H\&E) staining. The tumor-node-metastasis (TNM) stage of these tumors were assigned according to the American Joint Committee on Cancer guidelines. This study was conducted in accordance with the declaration of Helsinki. Written informed consent was obtained from all participants.

Inclusion criteria. This study was reviewed and approved by the Institutional Review Board and Ethics Committee of Zhengzhou University. The prospective subject cohort consisted of matched pairs of tumor/normal gastric tissues. The inclusion criteria are as follows: i) histological diagnosis of GC, ii) the tumor can be at any TNM stage, iii) gastric resection must have been performed with a curative/radical intention, and iv) signed informed consent was obtained; and v) no chemotherapy or radiotherapy treatment prior to surgery.

Proteomic chip-based cytokine antibody assay in GC tissues. All samples were used to assess the expression levels of 507 cytokines using 16 antibody arrays (RayBio ${ }^{\circledR}$ L-Series human antibody array L-507 membrane kit; RayBiotech, Norcross, GA,
USA) according to the manufacturer's instructions. Briefly, all protein samples were extracted from the tissues, followed by quantification and biotinylation. Incubation of the array of membranes with biological samples overnight at $4^{\circ} \mathrm{C}$ resulted in the binding of cytokines to corresponding antibodies. Signals were visualized using HRP-conjugated streptavidin and imaged by ImageQuant LAS 4000 Scanner (GE Healthcare Corp., Logan, UT, USA). The final spot intensities were measured as the original intensities, subtracting the background. The data were normalized to the positive controls in the individual slide and intensity ratios between GC and adjacent noncancerous mucosa tissues of each experiment were compared.

Bioinformatics analysis of differentially expressed protein factors associated with GC. The significant differentially expressed protein factors in the GC samples were analyzed using bioinformatics (http://www.expasy.org/vg/index/protein). Gene Ontology (GO) and Kyoto Encyclopedia of Genes and Genomes (KEGG) enrichment analysis of these protein factors were further conducted, aiming to screen the functions of the candidate cytokines associated with the development of GC.

Statistical analysis. All statistical and data analyses were performed using version 19.0 of the SPSS software (SPSS, Inc., Chicago, IL, USA). The P-values were calculated using a two sample t-test. In addition, fold change values of cytokines were calculated to indicate their relative expression levels normalized to the control samples. Any fold change $>2$ or $<0.5$ in signal intensity among the groups was considered relevant. $\mathrm{P}<0.05$ was considered statistically significant.

\section{Results}

Analysis of cytokines in GC. Analyses of 507 cytokines were made by the Tool Software for RayBio (RayBiotech, 


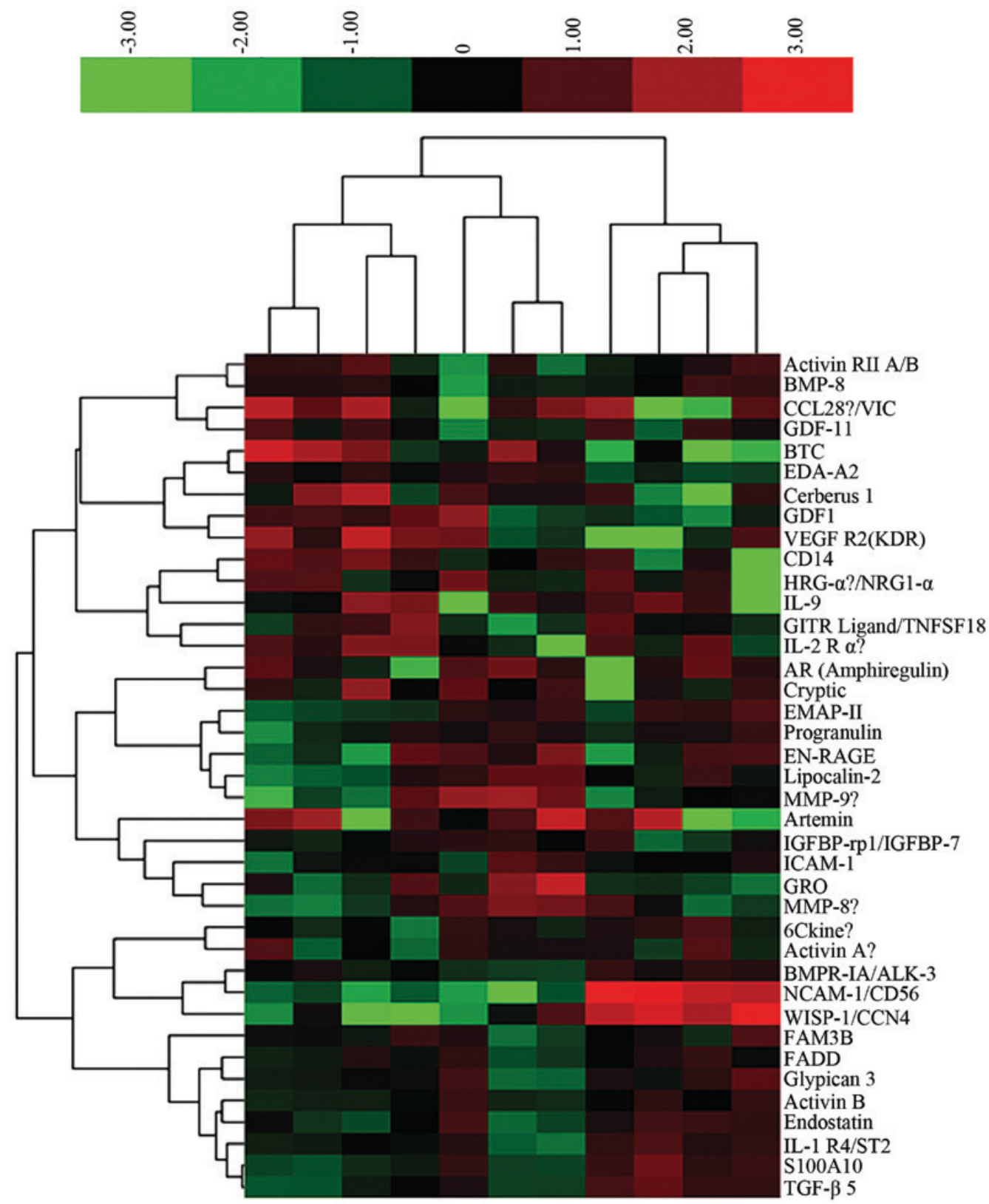

Figure 2. A 39-protein signatures that discriminates gastric cancer (GC) from non-GC tissues. To discover differences in protein abundance between samples of GC and those of non-GC, normalized array measurements in the training set was analyzed. Patient data were arranged in columns, and the proteins are listed in rows. High abundance ( $>2.0$-fold), no-change (between 2-fold and $>-2$-fold); low abundance (<-2-fold).

Inc., Norcross, GA, USA) human biotin-label based antibody arrays. All of the cytokines that were expressed in a given GC tissue were placed into the following groups based on their intensities relative to the non-GC tissue (Fig. 1): High abundance ( $>2.0$-fold), no-change (between 2-fold and $>$-2-fold), and low abundance (<-2-fold). These identifications were grouped in the heat map is shown in Fig. 2. One hundred and five differentially expressed proteins were identified in the samples (Table I).

Bioinformatics analysis of identified differentially expressed proteins in $G C$. In order to make high-throughput annotations of each protein and to determine the biological or functional distributions of differentially expressed cytokines, the GO and KEGG enrichment analysis of significantly differentially expressed proteins of each group were analyzed. As shown in Figs. 3-5, the specific biological processes or molecular functions that the candidate cytokines are involved in were determined, the degree of concordance between the differentially expressed cytokines and the expected functions are shown. Some proteins were found associated with certain functions (Table I).

\section{Discussion}

In recent years, despite some advancement of GC diagnosis, patients with GC still need to be diagnosed through invasive procedures such as endoscopy or surgery pathological diagnosis. Due to a low early diagnosis rate, most GC patients that are eventually diagnosed have already entered into the late stage with metastasis of the cancer, resulting a in low survival rate. The traditional biomarkers of GC, such as CEA, CA19-9 and 







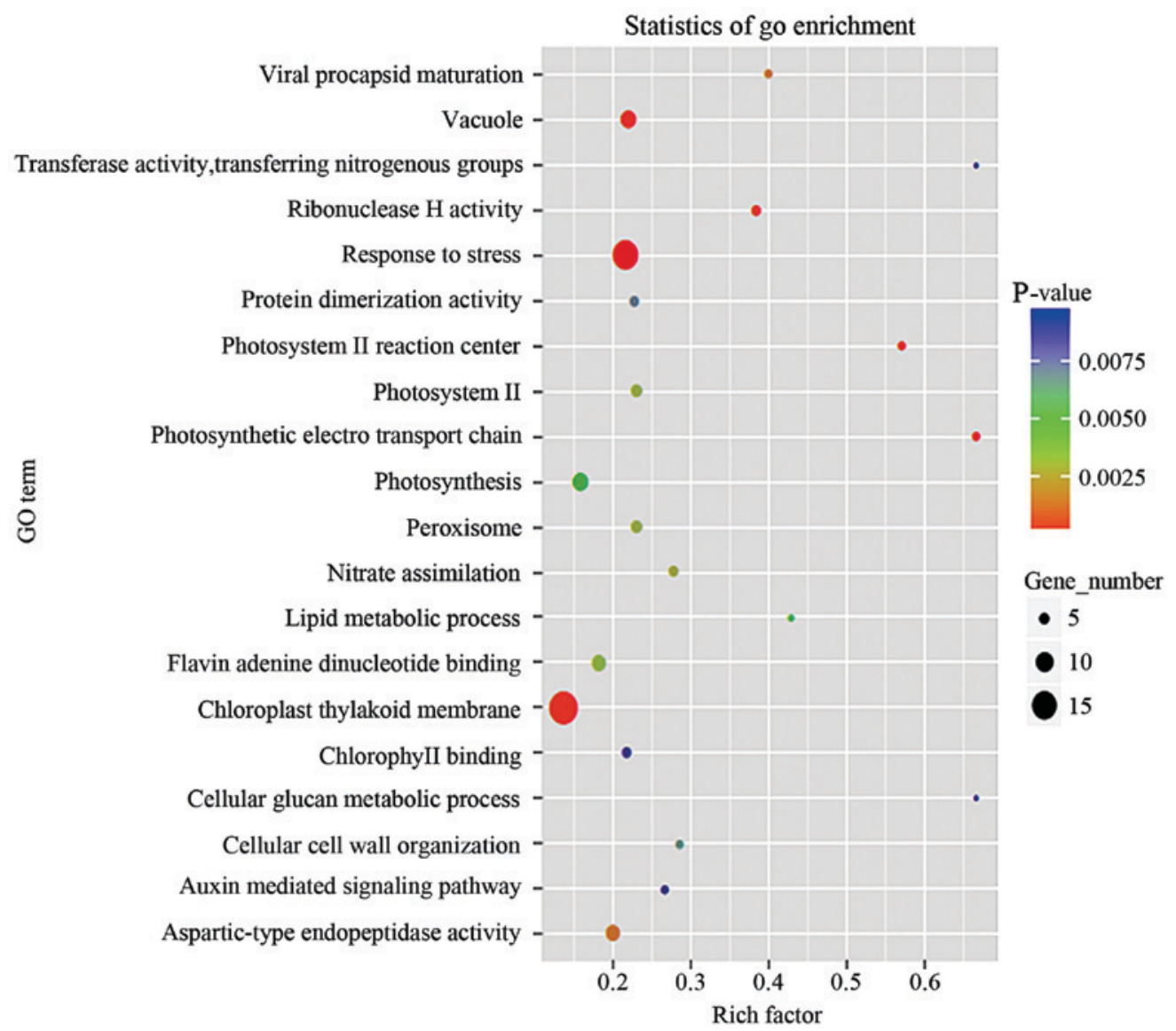

Figure 3. Gene Ontology (GO) enrichment analysis of differentially expressed proteins in gastric cancer (GC).

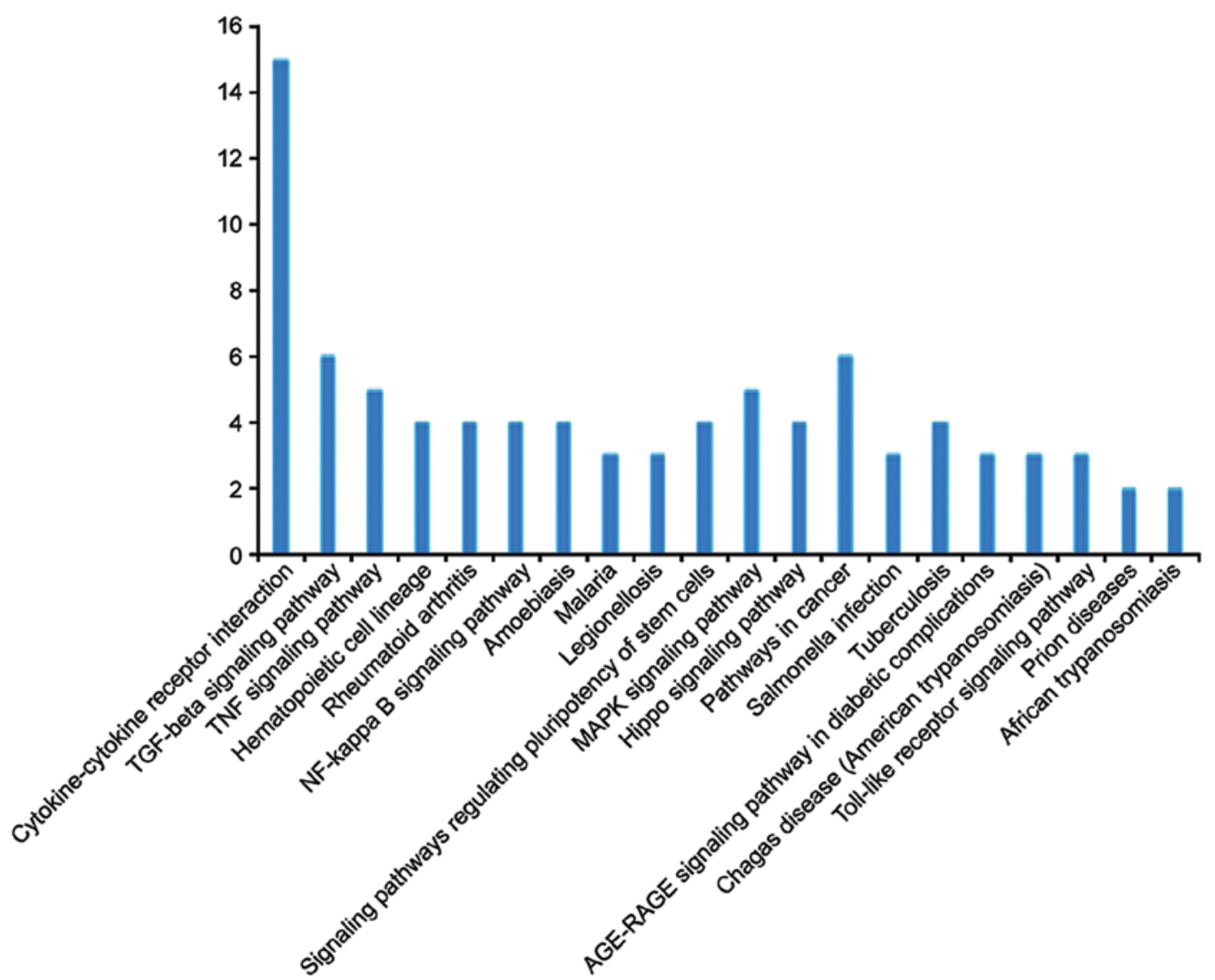

Figure 4. Kyoto Encyclopedia of Genes and Genomes (KEGG) enrichment analysis of differentially expressed proteins in gastric cancer (GC). 
$\mathbf{A}$

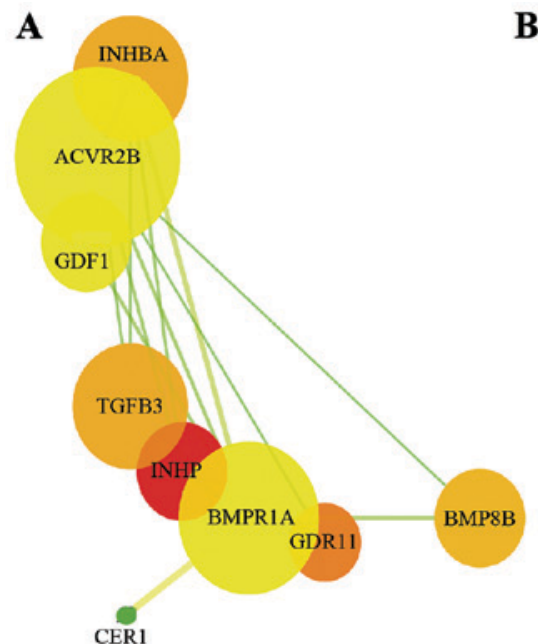

D



B

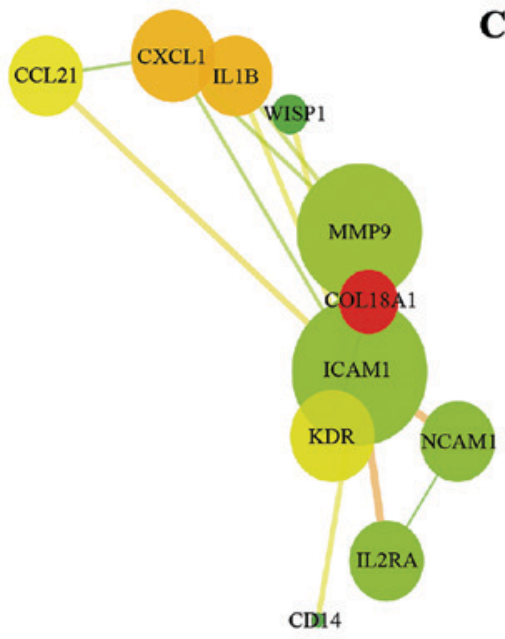

C

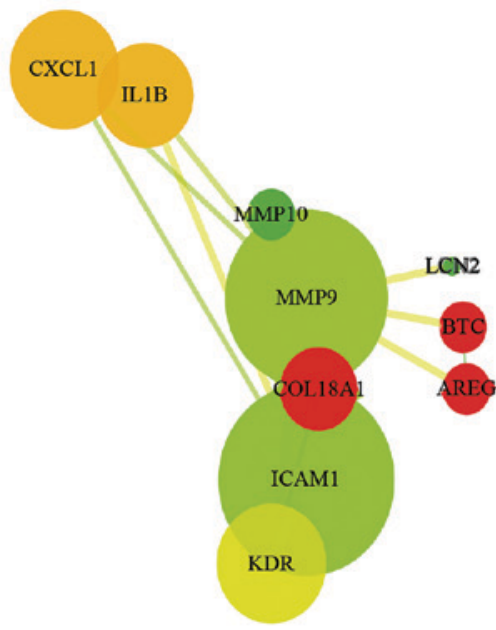

Figure 5. A functional analysis of 39 protein signatures. Network analysis of differentially expressed proteins included in INPROGAS. A dataset containing the differentially expressed biomarkers in gastric cancer (GC) tissues (called the focus molecules, $\mathrm{n}=39$ ) was overlaid onto a global molecular network developed from information contained in the IPA Knowledge Base.

XA74-4, are usually not specific and sensitive, as their sensitivity is only $18-57 \%$ (11). Therefore, looking for highly specific and sensitive GC biomarkers can contribute to early diagnosis, targeted therapy and a better prognosis of patients with GC (12).

Antibody microarray-based technology, which can simultaneously detect the expression levels of multiple proteins and has the combined advantages of the specificity of enzyme-linked immunosorbent assay (ELISA), sensitivity of enhanced-chemiluminescence (ECL) and high-throughput capacity of microspot, represents a promising tool for the field of onco-proteomics $(10,13)$. This assay can be used to compare and analyze proteins at various stages in the occurrence and development of tumors in order to screen for biomarkers for early diagnosis of tumors, specialized drug therapy and prognostic evaluation. In addition, this technology utilizes proteomics through the antibody microarray-based technology, which plays an important role in the research of clinical, pharmacology, signal transduction, cell cycle regulation, cell structure and neural biology $(14,15)$. Therefore, in the present study, we performed a proteomic chip-based analysis to investigate the differentially expressed cytokines that are associated with the development of GC. This assay is capable of rapidly 
and specifically detecting the expression levels of numerous cytokines, growth factors, soluble receptors of growth factors, angiogenic factors, metalloproteinases and other proteins using small amounts of experimental sample in a single experiment.

Chemokines and their receptors have shown a variety of biological functions in many processes, including the regulation of tumor cell proliferation, angiogenesis, invasion and metastasis (16-18). Many cancer-related cytokines, chemokines, metalloproteinases, growth factors and angiogenic factors are produced not only by the tumor cells themselves but also by the activated stroma and immune cells that are associated with tumors (16). The inflammatory mediators that are produced by immunocompetent cells and cancer cells can directly stimulate carcinogenesis (19-23).

As inflammation is a characteristic feature of the development and progression of GC, we hypothesized that cytokines released by the tumor microenvironment or by the cancer cells could represent novel diagnosis and predictive biomarkers. The simultaneous detection of multiple cytokines, which is afforded by this technology, is an important tool for biomarker discovery and can help us identify the key molecules that are important in cancer development (24). GO enrichment analysis showed that these significantly differentially expressed proteins in GC samples are involved in many biological and immunological processes, mainly in response to stress, chloroplast thylakoid membrane, vacuole, photosynthesis, aspartic-type endopeptidase activity and flavin-adenine dinucleotide binding. KEGG enrichment analysis demonstrated that these differentially expressed proteins are mainly involved in the process of cytokine-cytokine receptor interaction, transforming growth factor- $\beta$ (TGF- $\beta$ ) signaling pathway, tumor necrosis factor (TNF) signaling pathway, and mitogen-activated protein kinase (MAPK) signaling pathway. Moreover, our analysis revealed the key signaling pathways or networks that are related to a set of biomarkers identified in the training set. We imported the list of these 39 proteins into the IPA software.

In conclusion, our results suggest that 105 cytokines are frequently expressed in GC tissues and may be involved in occurrence and development of GC. While promising, our results are based on a relatively small sample of patients. A larger patient cohort is needed to validate the association of the candidate cytokines we identified and their involvement in GC. Further functional study of these cytokines may provide a promising approach for diagnostic and predictive biomarkers for GC.

\section{References}

1. Siegel R, Naishadham D and Jemal A: Cancer statistics, 2013. CA Cancer J Clin 63: 11-30, 2013.

2. Li NM, Liu F, Lv FY and Zhang QW: Influencing factors and interventional strategies for early enteral nutrition after gastric carcinoma surgery. J Cancer Res Ther 12: 689-692, 2016.

3. Fu G, Niu Z, Zhou Y, Zhou X, Wang H and Su Z: Influence of visceral fat area on laparoscopic radical gastrectomy in patients with gastric carcinoma. Zhonghua Wei Chang Wai Ke Za Zhi 18: 804-807, 2015 (In Chinese).
4. Peddanna N, Holt S and Verma RS: Genetics of gastric cancer. Anticancer Res 15: 2055-2064, 1995.

5. Sakai N, Yoshidome H, Shida T, Kimura F, Shimizu H, Ohtsuka M, Takeuchi D, Sakakibara M and Miyazaki M: CXCR4/CXCL12 expression profile is associated with tumor microenvironment and clinical outcome of liver metastases of colorectal cancer. Clin Exp Metastasis 29: 101-110, 2012.

6. Matboli M, El-Nakeep S, Hossam N, Habieb A, Azazy AE, Ebrahim AE, Nagy Z and Abdel-Rahman O: Exploring the role of molecular biomarkers as a potential weapon against gastric cancer: A review of the literature. World $\mathbf{J}$ Gastroenterol 22: 5896-5908, 2016.

7. Amedei A, Prisco D and D' Elios MM: The use of cytokines and chemokines in the cancer immunotherapy. Recent Pat Anticancer Drug Discov 8: 126-142, 2013.

8. Abdiche YN, Miles A, Eckman J, Foletti D, Van Blarcom TJ, Yeung YA, Pons J and Rajpal A: High-throughput epitope binning assays on label-free array-based biosensors can yield exquisite epitope discrimination that facilitates the selection of monoclonal antibodies with functional activity. PLoS One 9: e92451, 2014.

9. Perlee LT, Christiansen J, Dondero R, Grimwade B, Lejnine S, Mullenix M, Shao W, Sorette M, Tchernev VT, Patel DD, et al: Development and standardization of multiplexed antibody microarrays for use in quantitative proteomics. Proteome Sci 2: 9, 2004.

10. Kloppot P, Selle M, Kohler C, Stentzel S, Fuchs S, Liebscher V, Müller E, Kale D, Ohlsen K, Bröker BM, et al: Microarray-based identification of human antibodies against Staphylococcus aureus antigens. Proteomics Clin Appl 9: 1003-1011, 2015.

11. Ebert MP and Röcken C: Molecular screening of gastric cancer by proteome analysis. Eur J Gastroenterol Hepatol 18: 847-853, 2006.

12. Erkan M, Reiser-Erkan C, Michalski CW, Kong B, Esposito I, Friess $\mathrm{H}$ and Kleeff $\mathrm{J}$ : The impact of the activated stroma on pancreatic ductal adenocarcinoma biology and therapy resistance. Curr Mol Med 12: 288-303, 2012.

13. Puig-Costa M, Codina-Cazador A, Cortés-Pastoret E, OliverasFerraros C,Cufí S, Flaquer S, Llopis-Puigmarti F, Pujol-AmadoE, Corominas-Faja $\mathrm{B}$, Cuyàs $\mathrm{E}$, et al: Discovery and validation of an INflammatory PROtein-driven GAstric cancer Signature (INPROGAS) using antibody microarray-based oncoproteomics. Oncotarget 5: 1942-1954, 2014.

14. Liu Y: Serum proteomic pattern analysis for early cancer detection. Technol Cancer Res Treat 5: 61-66, 2006.

15. Ménard C, Johann D, Lowenthal M, Muanza T, Sproull M, Ross S, Gulley J, Petricoin E, Coleman CN, Whiteley G, et al: Discovering clinical biomarkers of ionizing radiation exposure with serum proteomic analysis. Cancer Res 66: 1844-1850, 2006.

16. Caronni N, Savino B, Recordati C, Villa A, Locati M and Bonecchi R: Cancer and chemokines. Methods Mol Biol 1393: 87-96, 2016.

17. Zhong J, Chen Y and Wang LJ: Emerging molecular basis of hematogenous metastasis in gastric cancer. World J Gastroenterol 22: 2434-2440, 2016

18. Verbeke H, Geboes K, Van Damme J and Struyf S: The role of CXC chemokines in the transition of chronic inflammation to esophageal and gastric cancer. Biochim Biophys Acta 1825: 117-129, 2012.

19. Biswas SK and Mantovani A: Macrophage plasticity and interaction with lymphocyte subsets: Cancer as a paradigm. Nat Immunol 11: 889-896, 2010.

20. Mariani F and Roncucci L: Chemerin/chemR23 axis in inflammation onset and resolution. Inflamm Res 64: 85-95, 2015.

21. Hanahan D and Weinberg RA: Hallmarks of cancer: The next generation. Cell 144: 646-674, 2011.

22. Galdiero MR, Garlanda C, Jaillon S, Marone G and Mantovani A: Tumor associated macrophages and neutrophils in tumor progression. J Cell Physiol 228: 1404-1412, 2013.

23. Jung M, Ören B, Mora J, Mertens C, Dziumbla S, Popp R, Weigert A, Grossmann N, Fleming I and Brüne B: Lipocalin 2 from macrophages stimulated by tumor cell-derived sphingosine 1-phosphate promotes lymphangiogenesis and tumor metastasis. Sci Signal 9: ra64, 2016.

24. Natesan M and Ulrich RG: Protein microarrays and biomarkers of infectious disease. Int J Mol Sci 11: 5165-5183, 2010. 